\title{
Microinjection of antibodies against talin inhibits the spreading and
}

\section{migration of fibroblasts}

\author{
GLEN H. NUCKOLLS ${ }^{1, *}$, LEWIS H. ROMER ${ }^{2}$ and KEITH BURRIDGE ${ }^{1,} \dagger$ \\ ${ }^{1}$ Department of Cell Biology and Anatomy, 108 Taylor Hall, CB no. 7090, and ${ }^{2}$ Department of Pediatrics, University of North Carolina at \\ Chapel Hill, Chapel Hill, North Carolina 27599-7090, USA
}

*Present address: Department of Biochemistry, Beckman Center, Rm 400, Stanford University School of Medicine, Stanford, CA 943055807 , USA

†Author for correspondence

\begin{abstract}
Summary
Talin is believed to be one of the key proteins involved in linking actin filaments to extracellular matrix receptors in focal adhesions. Our strategy for studying the function of talin has been to inactivate talin in living fibroblasts in tissue culture through the microinjection of affinity-purified, polyclonal anti-talin antibodies. The effect of the injected anti-talin antibodies on cell spreading was found to depend on how recently the cells had been plated. Cells that were in the process of spreading on a fibronectin substratum, and which had newly developed focal adhesions, were induced to round up and to disassemble many of the adhesions. However, if fibroblasts were allowed to spread completely before they were microinjected with the anti-talin antibody, focal adhesions remained intact and the flat morphology of the cells was unaffected. The percentage of cells that
\end{abstract}

were able to maintain a spread morphology despite the injection of anti-talin antibodies increased during the first few hours after plating on fibronectin substrata. Fibroblasts that were allowed to spread completely before microinjection with the anti-talin antibody retained both intact focal adhesions and a flat, wellspread morphology, but failed to migrate effectively. Our experiments do not directly address the role of talin in mature focal adhesions, but they indicate that talin is essential for the spreading and migration of fibroblasts on fibronectin as well as for the development and initial maintenance of focal adhesions on this substratum.

Key words: focal adhesions, vinculin, microinjection, talin, migration.

\section{Introduction}

Cells in culture typically adhere to the substratum through two different types of adhesion, known as focal adhesions (focal contacts) and close contacts (Izzard and Lochner, 1976). These two types of adhesion can be distinguished using interference reflection microscopy (IRM). Focal adhesions appear as discrete areas that are dark gray or black, indicating a separation between the ventral plasma membrane and the substratum of 10$15 \mathrm{~nm}$, whereas close contacts appear as broad regions that are lighter gray and represent a separation of about $30 \mathrm{~nm}$. At their cytoplasmic face, focal adhesions are associated with the ends of bundled actin filaments that make up stress fibers, whereas close contacts are associated with cross-linked networks of actin filaments (Heath and Dunn, 1978). Tension generated by stress fibers is transmitted across the plasma membrane to the substratum at sites of focal adhesion, and proteins that concentrate at these sites may serve as links between actin filaments and extracellular matrix (ECM) com- ponents (for review see Burridge et al., 1988). In contrast, little is known about the organization or composition of close contacts. Functionally, close contacts have been correlated with migratory activity and they are more prominent in rapidly locomoting cells (Couchman and Rees, 1979; Kolega et al., 1982).

Talin is a cytoskeletal protein that is concentrated in focal adhesions (Burridge and Connell, 1983; Hock et al., 1989) along with a number of other proteins. Various interactions between focal adhesion proteins in vivo have been proposed, based on the results of in vitro binding studies. For example, talin binds to ECM receptors of the integrin family (Horwitz et al., 1986), to the focal adhesion protein vinculin (Otto, 1983; Wilkins et al., 1983; Burridge and Mangeat, 1984) and also to actin (Muguruma et al., 1990). These interactions suggest that talin may function either as a direct link between integrins and actin or indirectly through its interaction with vinculin, which in turn has been shown to bind the actin-binding protein $\alpha$-actinin (Belkin and Koteliansky, 1987; Wachsstock et al., 1987). However, 
other potential linkages are also suggested by in vitro binding experiments. $\alpha$-Actinin, for example, has also been shown to bind to integrins directly (Otey et al., $1990 \mathrm{~b}$ ); and tensin has been reported to bind both actin and vinculin (Wilkins et al., 1987). The results of in vitro binding experiments provide important clues to both the interactions and the functions of these proteins inside cells. Nevertheless, confirming these functions will be difficult. We have performed three distinct assays to analyze the function of talin in living fibroblasts cultured on fibronectin. We have microinjected anti-talin antibodies into well-spread fibroblasts with mature focal adhesions. We have injected the antibodies into spreading fibroblasts that had only newly formed focal adhesions, and we have injected the antibodies into fibroblasts with mature focal adhesions that were induced to migrate by the wounding of a confluent monolayer. We found that the microinjection of these antibodies could inhibit fibroblast migration and spreading, two processes that involve the formation of new focal adhesions. Furthermore, we found that the antibody could disrupt recently formed focal adhesions and stress fibers.

\section{Materlals and methods}

\section{Cell culture}

Chicken embryo dermal fibroblasts (CEF) were isolated from 8- to 12-day-old embryos as described previously (Duband et al., 1988). CEF were grown in Dulbecco's modified Eagle's medium (DMEM) containing 10\% fetal bovine serum (FBS), $15 \mathrm{mM}$ HEPES (pH 7.4), $100 \mathrm{i} . \mathrm{u} . / \mathrm{ml}$ penicillin and $100 \mu \mathrm{g} / \mathrm{ml}$ streptomycin (pen/strep) in a $37^{\circ} \mathrm{C}, 5 \% \mathrm{CO}_{2}$ incubator.

\section{Affinity purification of anti-talin antibodies and preparation of control antibodies}

Approximately $10 \mathrm{mg}$ of talin, purified from chicken gizzard as described by Molony et al. (1987), was dialyzed overnight against a buffer of $20 \mathrm{mM} \mathrm{NaCl}, 20 \mathrm{mM}$ MES, $\mathrm{pH} 7.4$. The talin was coupled to $2 \mathrm{ml}$ Affigel 10 (BioRad, Richmond, CA) following directions supplied by the manufacturer. Unreacted sites on the Affigel were blocked with ethanolamine. The gel was washed extensively with $\mathrm{PBS}^{-}$(phosphate buffered saline without $\mathrm{Ca}^{2+}$ or $\mathrm{Mg}^{2+}$ ) plus $0.1 \% \mathrm{NaN}_{3}$. Approximately $3 \mathrm{ml}$ of rabbit anti-talin serum (characterized by Rochlin et al., 1989) was centrifuged at $15,000 \mathrm{~g}$ for $15 \mathrm{~min}$ and the supernatant was diluted $1: 1$ with PBS $^{-}$plus $\mathrm{NaN}_{3}$ before incubating with the talin-Affigel for $4 \mathrm{~h}$ at $4^{\circ} \mathrm{C}$. After the incubation, the gel was packed by centrifugation at $300 \mathrm{~g}$ for 3 $\min$ and the supernatant was removed.

The antiserum that was applied to the talin-Affigel beads was routinely used to immunostain fibroblasts at a dilution of 1:5000; however, the supernatant from the talin-Affigel beads gave no focal contact staining on CEF at a dilution of 1:10. The IgG in this supernatant was used for control microinjections after it was concentrated by ammonium sulfate precipitation and dialyzed against antibody injection buffer $(75 \mathrm{mM}$ $\mathrm{KCl}, 10 \mathrm{mM}$ potassium phosphate buffer, $\mathrm{pH}$ 7.5).

The talin affinity column with antibody bound was washed with 50 bed volumes of $\mathrm{PBS}^{-}$with $0.1 \% \mathrm{NaN}_{3}$ and then bound antibody was eluted with $100 \mathrm{mM}$ triethylamine, $\mathrm{pH}$ 11.5 , followed by a wash with 10 bed volumes of PBS $^{-}$. After washing, the column was further eluted with $100 \mathrm{mM}$ glycine-
$\mathrm{HCl}, \mathrm{pH}$ 2.6. Peak fractions from each of these elutions were pooled and concentrated by dialysis against sucrose, followed by extensive dialysis against antibody injection buffer. The final sample of affinity-purified anti-talin was determined to be $3 \mathrm{mg} / \mathrm{ml}$ by spectrophotometric measurements. At a dilution of $1: 10,000$, this antibody stained a typical focal adhesion pattern in fibroblasts (data not shown).

\section{Antibody microinjection}

Coverslips were incubated with $50 \mu \mathrm{g} / \mathrm{ml}$ human plasma fibronectin (New York Blood Center, New York) in PBS $^{+}$ (PBS with $\mathrm{Ca}^{2+}$ and $\mathrm{Mg}^{2+}$ ) for $30 \mathrm{~min}$ at $37^{\circ} \mathrm{C}$ followed by a brief wash with $\mathrm{PBS}^{+}$. CEF, after several passages in tissue culture were plated onto these coated coverslips in complete medium. Depending on the experiment, CEF plated onto fibronectin-coated coverslips were incubated for $15 \mathrm{~min}$ to 12 $h$ (pre-injection incubation). CEF used for migration experiments were cultivated on fibronectin-coated coverslips for a 24-h pre-injection incubation, and the entire CEF population was then removed from one half of the slide with a cell scraper. At the end of the pre-injection incubation, the coverslips for all experiments were transferred to warm, gassed, fresh medium for microinjection.

Cells were microinjected using the method described by Graessmann et al. (1980). Experimental and control injections were performed on the same coverslip. A diamond scribe was used to scratch a circle and a square on each coverslip. Cells within the circle were microinjected with affinity-purified anti-talin antibodies, and cells within the square with control antibodies all using essentially identical Kwik-fil glass capillaries (World Precision Instruments, New Haven, CT) pulled on a Brown-Flaming micropipette puller (Sutter Instrument Co., San Francisco, CA). The length of the needles was minimized to prevent loss of antibody due to adsorption to the glass during loading; most needles were approximately $2 \mathrm{~cm}$ in length. Microinjection was performed on a Leitz Diavert microscope using a $\times 32$ phase-contrast objective and a Leitz micromanipulator. After the injections, the coverslips were returned to the incubator for a 2- to 3-h post-injection incubation or in one experiment that tested the cells' ability to recover from the anti-talin antibody microinjection there was a 43-h incubation.

For the migration experiments, experimental and control injections were performed on separate, paired coverslips. Only the cells immediately adjacent to the wound edge created by the cell scraper were injected. These coverslips then received a 12-h post-injection incubation to allow recovery and migration into the denuded one half of the coverslip.

\section{Immunostaining and microscopy}

Coverslips were fixed prior to permeabilization and immunostaining using protocols described previously (Nuckolls et al., 1990). Immunostaining for talin was performed using either a monoclonal, mouse anti-talin antibody characterized previously (Otey et al., 1990a), or the rabbit anti-talin serum used for affinity preparations. Immunostaining of vinculin was accomplished using a monoclonal anti-chicken vinculin antibody that was developed in this laboratory. Secondary antibodies were an FITC-conjugated, species cross-adsorbed, donkey anti-rabbit IgG (Jackson Immuno-research Lab., West Grove, PA) diluted in $\mathrm{PBS}^{+}$to $1: 100$ or a TRITCconjugated goat anti-mouse IgG (Cappel, Malvern, PA) diluted in $\mathrm{PBS}^{+}$to $1: 50$. Actin stress fibers were stained using rhodamine-conjugated phalloidin (Molecular Probes, Eugene, OR). After a final wash, the coverslips were rinsed in deionized water and mounted in $10 \%$ Mowiol (Calbiochem, 
San Diego, CA) in a Tris buffer, pH 8.5, and air dried. For interference reflection microscopy (IRM), the well of a hanging-drop mount slide was filled with $50 \%$ glycerol in PBS $^{-}, 0.1 \% \mathrm{NaN}_{3}, \mathrm{pH}$ 8.5. The coverslips were inverted and mounted in the glycerol solution and sealed with silicon grease. Fluorescence images were photographed onto TMAX 400 or P3200 film with Zeiss Planapo $\times 63$ or Plan-Neofluar $\times 40$ or $\times 20$ objective lenses. IRM images were photographed through a Zeiss Antiflex $\times 63$ objective on Kodak Technical Pan 2415 film developed with D19.

To verify that cells had survived the microinjection, selected coverslips were incubated with $5 \mu \mathrm{M}$ propidium iodide in DMEM prior to fixation according to the method described by Lemasters et al. (1987). After viewing the areas of the coverslip that had been microinjected with anti-talin or control antibodies, any cells that had taken up the vital stain were photographed, and then these coverslips were fixed and immunostained to identify the cells that contained injected antibody.

\section{Results}

\section{Injection of fully spread fibroblasts with anti-talin}

CEF were plated on glass coverslips $12 \mathrm{~h}$ prior to microinjection. Cells on the coverslip were injected with affinity-purified, polyclonal rabbit anti-chicken talin antibodies at a concentration of $3 \mathrm{mg} / \mathrm{ml}$ or with control antibodies at equal or higher concentrations. Injections were estimated to be $10-20 \%$ of the volume of the cell (about $10^{-7} \mu \mathrm{l}$ injected per cell). The coverslips were incubated for $3 \mathrm{~h}$ after the injection to allow for the antibodies to gain access to all parts of the injected cells. The cells were then fixed and immunostained with a fluorescent anti-rabbit antibody to localize the injected antibody, and vinculin or talin was localized in the same cells using monoclonal antibodies and indirect immunofluorescence. Interference reflection microscopy (IRM) and immunostaining demonstrated that anti-talin antibodies injected into fibroblasts decorated structures containing talin. Fig. 1 demonstrates the localization of anti-talin antibodies microinjected into a well-spread fibroblast. The injected antibody localized to focal adhesions (arrow in Fig. 1B) that were also sites of concentration of the protein vinculin (arrow in Fig. 1C). The corresponding IRM image (Fig. 1A) demonstrated that focal adhesions appeared normal and a well-spread morphology was maintained in these cells. The injected antibody also labelled a fibrillar pattern that is only weakly labelled with antibodies against vinculin. This pattern is similar to the distribution of talin in many cells when talin aligns with ECM components such as fibronectin (Burridge and Connell, 1983). Injected cells continued to display normal patterns of stress fibers (data not shown). Control injected antibodies were distributed diffusely throughout the cytoplasm and were excluded from the nucleus (see Fig. 3B, below).

\section{Anti-talin injected into spreading cells}

Since the injection of anti-talin did not perturb fibroblasts that were completely spread and had mature adhesions, we investigated whether the antibody would affect cell spreading. Prior to these experiments we evaluated the spreading of CEF on different substrata: untreated glass coverslips, serum-treated coverslips and coverslips coated with fibronectin. Spreading was fastest and most consistent on the fibronectin-coated coverslips (our unpublished results). Consequently, we used fibronectin-coated coverslips for all the spreading experiments. CEF were trypsinized, washed and plated onto fibronectin-coated coverslips. Cells that have been
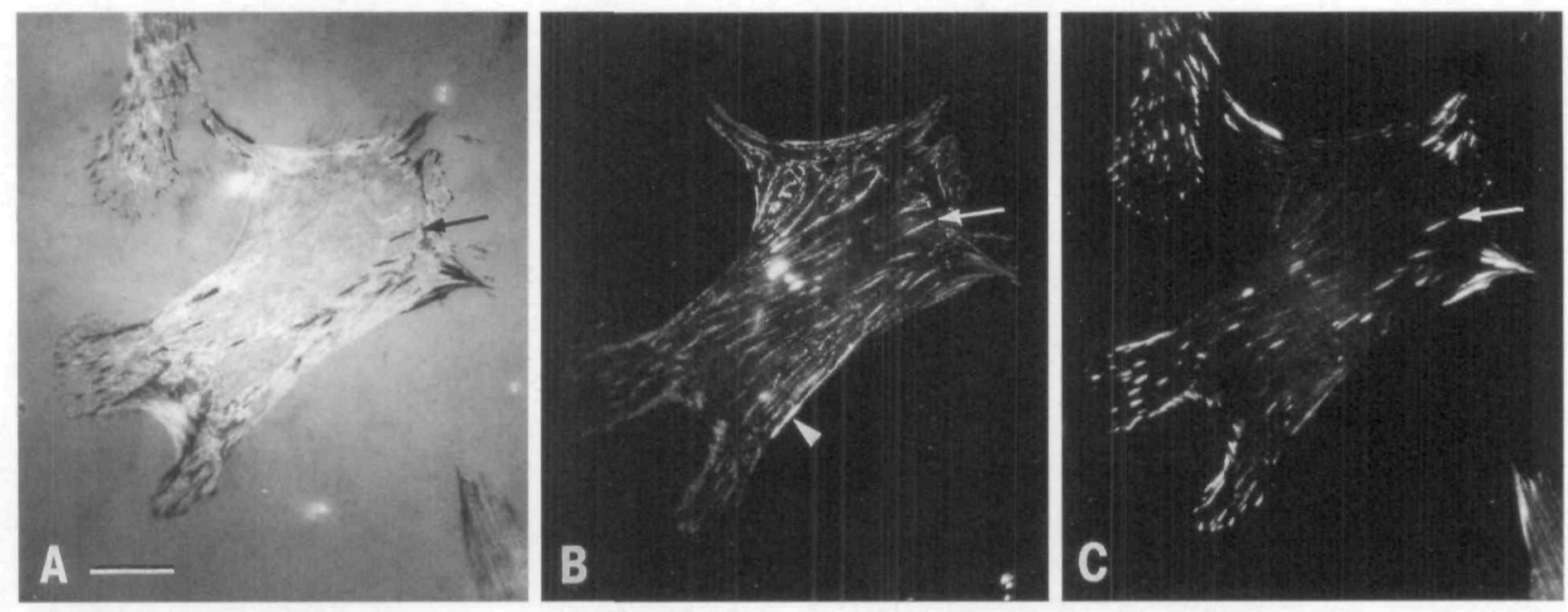

Fig. 1. Microinjection of anti-talin antibodies into well-spread fibroblasts. CEF were plated onto coverslips for $12 \mathrm{~h}$ and then microinjected with the anti-talin antibody. The cells were fixed $2 \mathrm{~h}$ after the microinjection and immunostained for the injected antibody (B) and for vinculin (C). A portion of a cell that has not been injected with the anti-talin antibody is seen in the upper left corner of two of the images. Cells displayed numerous focal adhesions (arrows) visualized by interference reflection microscopy (A), which are characteristic of well-spread CEF. The injected antibodies concentrated at these sites but did not appear to disrupt them. The injected antibody also localized in fibrillar streaks that stain weakly for vinculin, and are not seen in the IRM image (arrowhead in B). Vinculin distribution appears unaffected by anti-talin injection. Bar, $20 \mu \mathrm{m}$. 

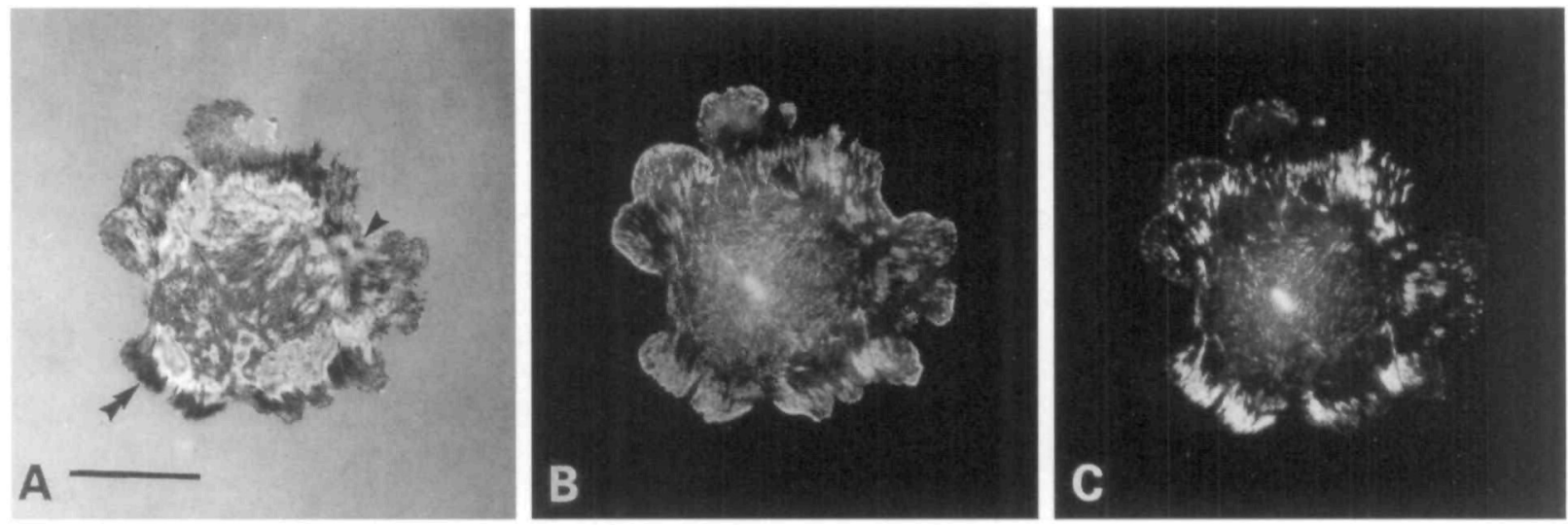

Fig. 2. Adhesions of fibroblasts during cell spreading. CEF were plated onto fibronectin-coated coverslips and incubated for $30 \mathrm{~min}$. The cells were then fixed and immunostained for talin (B) and vinculin (C). The IRM image (A) reveals small, dot-like focal adhesions (arrowhead), as well as broad areas of focal adhesion (double arrowhead). Talin is concentrated in focal adhesions and at the cell's margin. Vinculin is concentrated in focal adhesions, but is less prominent than talin at the cell's margin. Bar, $20 \mu \mathrm{m}$.

spreading for $30 \mathrm{~min}$ on fibronectin-coated coverslips exhibit several structures that contribute to the adhesions of these cells (Fig. 2). The IRM image (Fig. 2A) shows small dot-like focal adhesions (arrowhead) as well as broad areas of focal adhesion (double arrowhead). Although there are some regions of close contact, the predominant adhesions are focal. Talin is concentrated in the focal adhesions, and is also found at the cell margin (Fig. 2B). Vinculin is concentrated in the focal adhesions, but, as described previously (Burridge and Connell, 1983; Izzard, 1988), it is not as prominent at the cell margin as talin (Fig. 2C).

Fig. 3A shows a sample field of CEF that were allowed to spread on fibronectin-coated coverslips for 30 min before injecting the cells with anti-talin antibody. After the 3-h post-injection incubation, the
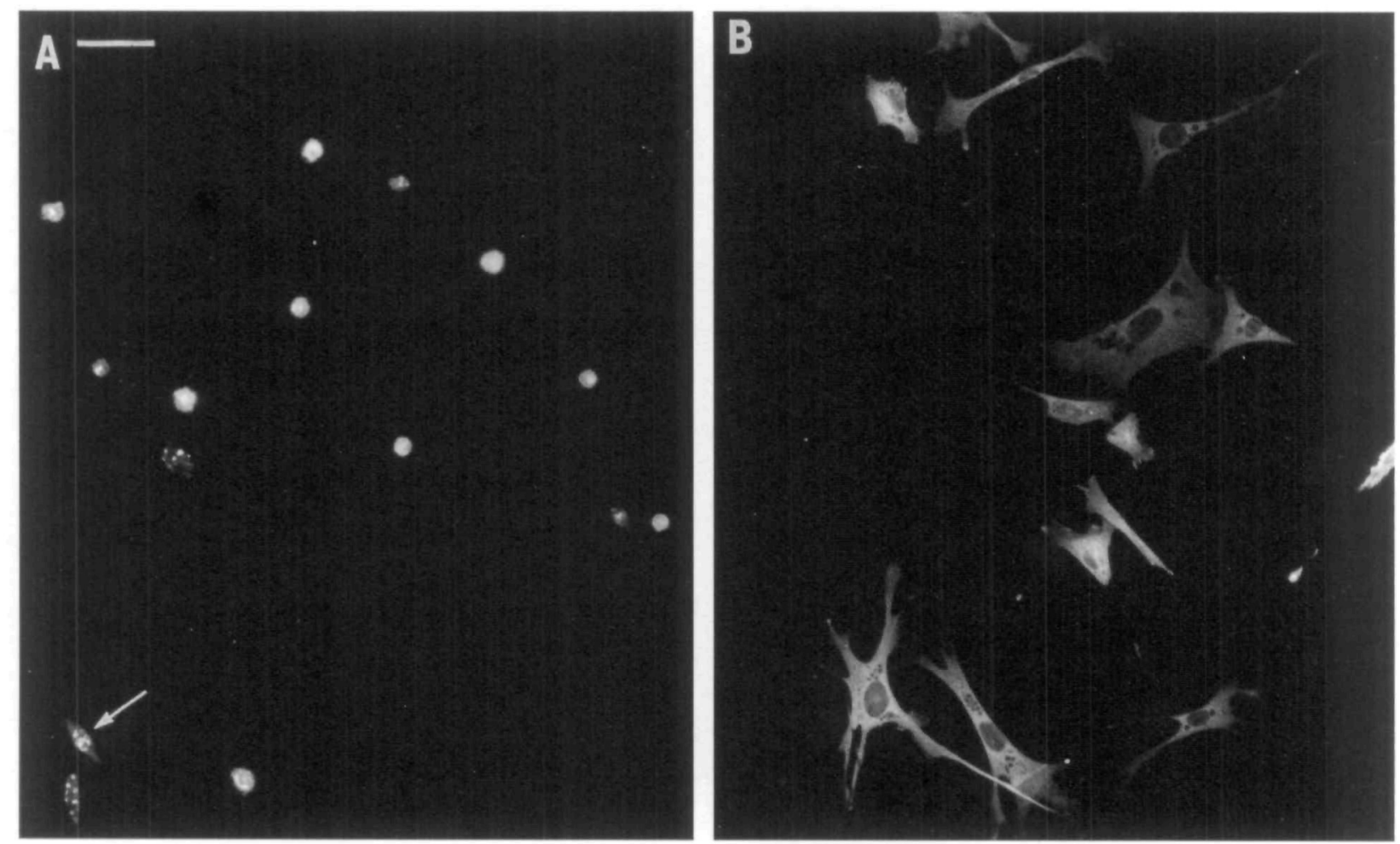

Fig. 3. The effects of the anti-talin antibody microinjection on spreading fibroblasts. After 30 min of spreading on fibronectin, CEF were microinjected with affinity-purified polyclonal anti-talin (A), or a control antibody (B). Two hours after the injection, cells were fixed and the microinjected antibodies were localized. The anti-talin antibody caused most cells to round up while control injected cells spread out and exhibited a normal fibroblast morphology. The arrow in A indicates a cell that has been affected by the anti-talin injection but, according to the criteria used, this cell would be classified as spread, since it has visible cell extensions. Bar, $50 \mu \mathrm{m}$. 

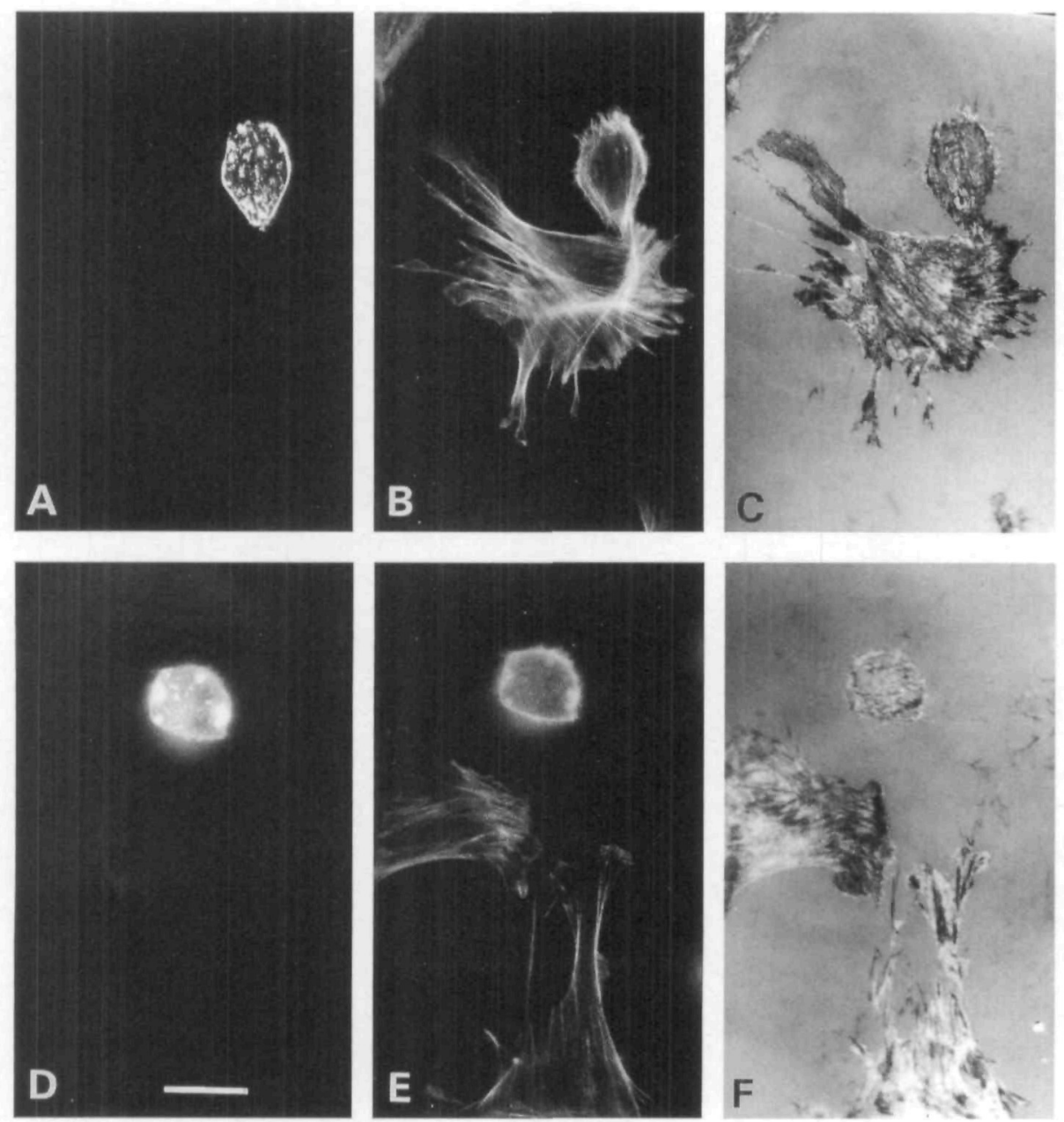

Fig. 4. Stress fibers and adhesions were perturbed by the injection of anti-talin antibodies into spreading fibroblasts. The localization of injected anti-talin antibodies $(\mathrm{A}$ and $\mathrm{D})$ shows extensive precipitates in the injected cells. Rhodamine-phalloidin staining ( $B$ and $E$ ) reveals a reduced number or absence of stress fibers in the injected cells. IRM images ( $\mathrm{C}$ and $\mathrm{F}$ ) demonstrate that the anti-talin injections have abolished most of the focal adhesions. Bar, $20 \mu \mathrm{m}$.

cells were fixed and a fluorescent secondary antibody was applied. The injection of anti-talin not only inhibited cell spreading, but reversed the spreading that had occurred during the $30 \mathrm{~min}$ of incubation prior to microinjection. In parallel, cells on the same coverslip were microinjected with a control antibody (Fig. 3B). Unlike cells injected with anti-talin, the cells injected with a control antibody spread out normally and displayed a morphology indistinguishable from noninjected cells. The cells injected with control antibodies contained normal stress fibers and formed focal adhesions that were sites of talin concentration (data not shown).

Examples of cells rounded by injection of talin antibodies are shown in Fig. 4 at higher magnification. These cells have been stained to visualize the injected antibody (Fig. 4A and D) and the distribution of actin (Fig. 4B and $\mathrm{E}$ ). The adhesions to the substratum have been visualized by IRM (Fig. 4C and F). The injected antibody is visible as brightly staining precipitates in both cells, but in Fig. 4D there is also a diffuse distribution of antibody superimposed on this pattern, probably indicating an excess of antibody in the cytoplasm and that this cell received relatively more than the cell in Fig. 4A. It is notable that this cell lacks any discernible stress fibers (Fig. 4E) and has very few adhesions visible by IRM (Fig. 4F). The cell in the top

panel shows a few residual adhesions and stress fibers, particularly at the periphery of the cell. It is interesting that the antibody is also concentrated along the margin of the cell coincident with these few stress fibers and some of the adhesions. In comparison with the noninjected cells in these images, however, this cell has diminished stress fibers and much smaller, less-prominent focal adhesions.

\section{Quantitation of the effects of anti-talin injection}

As was shown in Fig. 1, CEF that had been adhering to coverslips overnight did not change their morphology when injected with anti-talin antibody, but cells that were in the process of spreading on coverslips became rounded by the injection of anti-talin (Fig. 3). To quantitate the effects of the microinjection of anti-talin throughout the period of focal adhesion formation and maturation, CEF were plated onto fibronectin-coated coverslips and given pre-injection incubations of $30 \mathrm{~min}$ to $12 \mathrm{~h}$. The cells in one area of each coverslip were microinjected with the anti-talin antibody, while cells in an adjacent area of each coverslip were injected with the control antibody. At the end of the post-injection incubation, the coverslips were fixed and the injected antibodies were immunolocalized. Using a low-magnification objective lens, fluorescence and phase-contrast 


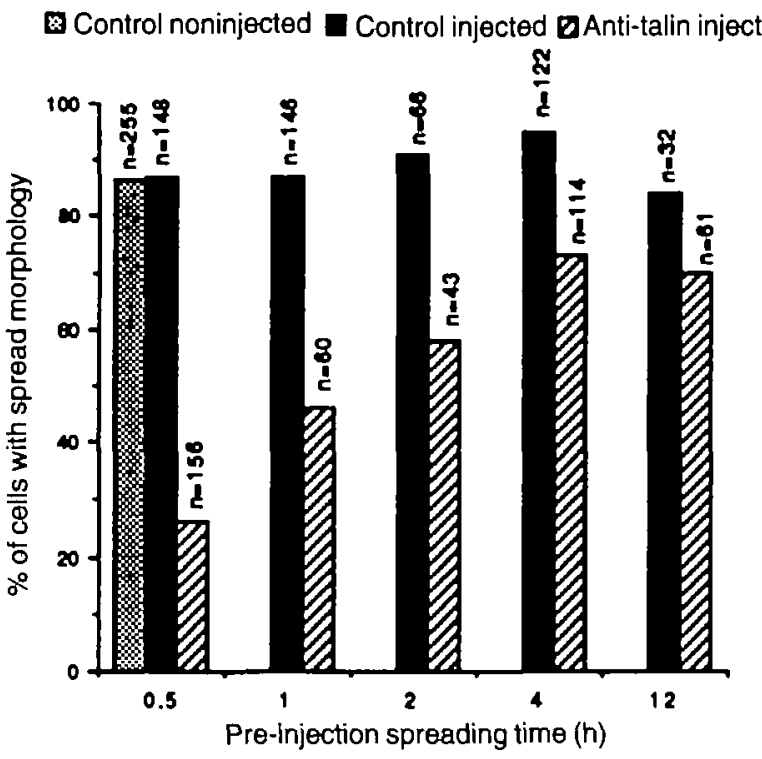

Fig. 5. Several hours after plating, CEF become resistant to the injected anti-talin antibody. CEF were plated onto fibronectin-coated coverslips and incubated for $30 \mathrm{~min}, 1 \mathrm{~h}$, $2 \mathrm{~h}, 4 \mathrm{~h}$ or $12 \mathrm{~h}$, and were then microinjected with the anti-talin antibody, or the control antibody. Experimental and control injections were performed on adjacent areas of the same coverslips. After a $3 \mathrm{~h}$ post-injection incubation, the cells were fixed and a fluorescent secondary antibody was applied. Phase-contrast and fluorescence microscopy images were compared, and each cell containing a detectable amount of injected antibody was classified as either round or spread.

images of relevant areas of the coverslips were compared. Although the morphologies of anti-talin- or control-injected cells varied, for the purpose of these experiments, cells containing the microinjected antibodies were classified simply as either round or spread, and the number of cells of each type of morphology was tallied. A cell was scored as spread if it displayed a thin lamella or if it had cell processes detectable at low magnification that were at least twice as long as the cell body. Cells lacking these structures were classified as round and were most often surrounded by a phase-light region. According to these criteria, at the time of microinjection essentially all cells were spread. Therefore, a rounded morphology at the end of the postinjection incubation indicates not only an inhibition of spreading, but a reversal of spreading in that cell.

The combined data from such experiments are shown in Fig. 5. In cells injected after a $30-\mathrm{min}$ pre-injection incubation, and examined $3 \mathrm{~h}$ later, $26 \%$ of the cells on the coverslip that contained detectable amounts of the anti-talin antibody $(n=156)$ displayed a spread morphology after the post-injection incubation. By comparison, $86.5 \%$ of cells containing detectable amounts of control antibody $(n=148)$ or $87 \%$ of non-injected cells on the same coverslip $(n=255)$ displayed a spread morphology after the post-injection incubation. In cells that were injected after an hour of spreading, the percentage of anti-talin-injected cells that were able to maintain the flat morphology increased to $46 \%(n=60)$.
The increased pre-injection incubation did not affect the morphology of control-injected cells, of which $88 \%$ were spread $(n=146)$. When the pre-injection incubation was increased to $2 \mathrm{~h}$, the percentage of anti-talininjected cells that displayed a spread morphology after the post-injection incubation increased to $58 \%(n=43)$. $91 \%$ of control injected cells $(n=66)$ were spread in this time course. By $12 \mathrm{~h}$ of pre-injection incubation, the morphology of anti-talin-injected cells approached that of control-injected, or non-injected cells.

It is important to note that cells displaying a rounded morphology are more likely to be lost from the coverslip during the process of fixation, permeabilization and immunostaining than cells that have a spread morphology. This phenomenon would tend to skew the percentages, and so the actual difference between control and experimental injected cells in experiments with short pre-injection incubations may be greater than that shown in Fig. 5.

\section{Assessment of cell viability}

CEF that had been plated on fibronectin-coated coverslips for $30 \mathrm{~min}$ were microinjected, incubated for $3 \mathrm{~h}$, and stained with the fluorescent vital dye propidium iodide. None of the rounded cells in the area of the coverslips that had been injected with the anti-talin antibody were able to take up the dye. This indicated that their plasma membranes were intact. The coverslips were then fixed and immunostained to localize the injected antibody, which revealed that round cells that contained talin immunoprecipitates were in fact cells that had excluded the propidium iodide (data not shown). There was a small percentage of dead, noninjected cells on each coverslip that took up the propidium iodide, and these cells served as controls for the vital staining.

Experiments were also performed to determine whether cells rounded by the injection of anti-talin antibodies could recover from these effects. Two hours after microinjecting anti-talin into all of the cells within a scored area of the coverslip, an empty microinjection needle was used to remove all of the spread cells within the scored area. This left only rounded cells in the area where cells had been injected with the anti-talin antibody. The coverslip was transferred to a dish containing fresh medium, and the cells were returned to the incubator for an extended post-injection incubation. At $43 \mathrm{~h}$ after microinjection, the coverslip was fixed and a fluorescent secondary antibody was applied. Fig. 6 shows a representative cell from the scored area of the coverslip. This cell shows some fibrillar staining and a dense aggregate of anti-talin (Fig. 6B). The IRM image of this cell that had been previously rounded with antitalin antibodies demonstrates that focal adhesions were formed during the 43-h post-injection period (Fig. 6C). Frequently the aggregate of antibody seen in the fluorescence images of these cells could also be detected in the phase-contrast images (arrowhead in Fig. 6A) and often there were phase-dense granules, probably lysosomes, associated with these aggregates. Cells microinjected with the control antibody continued to 

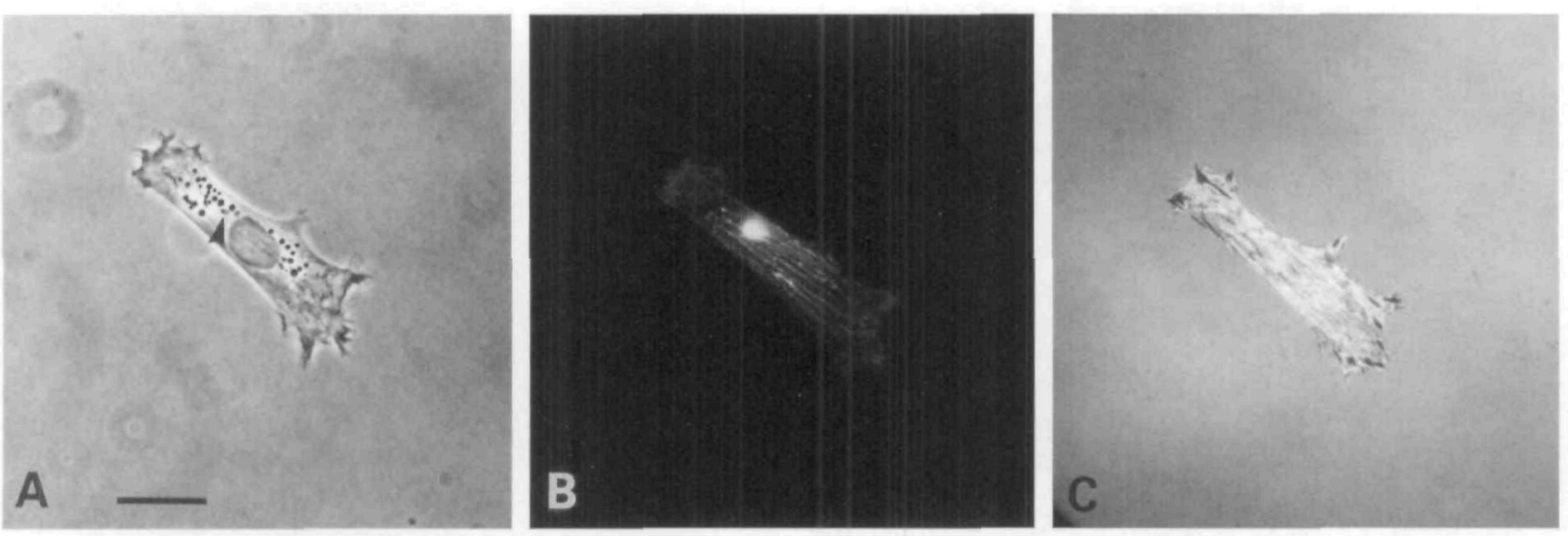

Fig. 6. Recovery from anti-talin injection. CEF that had been injected with the anti-talin antibody and that were known to have been rounded $2 \mathrm{~h}$ after the injection were incubated for an additional $41 \mathrm{~h}$, and then fixed and stained for the injected antibody. An aggregate of anti-talin was seen in most cells (B), and this aggregate could usually be correlated with a dense structure (arrowhead) in the phase-contrast image (A) close to the nucleus. The IRM image (C) demonstrates that new focal adhesions formed during the post-injection incubation. Bar, $20 \mu \mathrm{m}$.

Table 1. Effects of anti-talin antibody on fibroblast migration

\begin{tabular}{lcccc}
\hline Antibody injected & $\begin{array}{c}\text { Total no. cells } \\
\text { injected (no. expts) }\end{array}$ & $\begin{array}{c}\text { Baseline } \\
(\%)\end{array}$ & $\begin{array}{c}\text { Migrating pack } \\
(\%)\end{array}$ & $\begin{array}{c}\text { Leading edge } \\
(\%)\end{array}$ \\
\hline Affinity-purified anti-talin & $195(7)$ & $158(81)$ & $37(19)$ & $0(0)$ \\
Control & $154(5)$ & $2(<2)$ & $71(46)$ & $81(53)$ \\
\hline
\end{tabular}

display a diffuse distribution of injected antibody after $43 \mathrm{~h}$ of post-injection incubation that was indistinguishable from the distribution of this antibody in equivalent cells $3 \mathrm{~h}$ after injection (Fig. 3B).

\section{Anti-talin effects on fibroblast migration}

Since the microinjection of anti-talin antibody affected focal adhesion formation in spreading fibroblasts, we studied the impact of anti-talin on fibroblast migration, another activity requiring the formation of adhesions. CEF were plated on fibronectin-coated coverslips at confluent density, and incubated in complete medium for $24 \mathrm{~h}$. The entire population of CEF was then cleared from one half of the coverslip with a plastic cell scraper, leaving a straight-edged wound margin to serve as a starting baseline for cell migration. Separate coverslips were then used to study the effects of either anti-talin antibody or control antibody on fibroblast migration by injecting cells that were immediately adjacent to the wound edge. Following a 12-h post-injection incubation, cells were fixed, permeabilized and immunostained with a fluorescein-conjugated anti-rabbit antibody to identify injected cells, as well as with rhodamine-conjugated phalloidin to visualize all of the cells on the coverslip. Injected cells were then counted and their migration behavior categorized with one of the following three descriptions: remaining at the starting baseline of the wound edge; leaving the wound edge to join the migrating pack of cells; or taking a position at the leading edge of the cells migrating into the denuded half of the coverslip. A complete tabulation of the data from the 349 cells injected in these experiments is summarized in Table 1 . In the group injected with anti-talin antibodies ( $n=195)$, only $19 \%$ of the injected cells left the baseline of the wound edge to join the migrating pack and none of these took a position at the leading edge of the migrating cell front. In the group injected with control antibodies $(n=154)$, less than $2 \%$ of the injected cells remained at the baseline of the wound edge, while $53 \%$ took positions at the leading edge of the migrating cell front, and the remaining $46 \%$ had joined the migrating pack. Representative fields from the anti-talin and control injection groups are shown in Fig. 7. The cell injected with anti-talin antibodies (Fig. 7B) has remained at the baseline of the wound edge (white arrows), and is left behind by the migrating noninjected cells in the same microscopic field that are stained with rhodamine-phalloidin (Fig. 7A). Cells injected with control antibodies (Fig. 7D) are shown migrating in positions both at the leading edge and well into the migrating pack and may be compared with noninjected cells stained with rhodamine-phalloidin in the same microscopic field (Fig. 7C).

\section{Discussion}

Talin is a prominent protein at the cytoplasmic face of focal adhesions. Like many of the other cytoplasmic components of focal adhesions, the function of talin has not been established. The interaction of talin with both integrins and actin in vitro (Horwitz et al., 1986; Muguruma et al., 1990) together with its location in focal adhesions, suggests a probable role for talin in linking actin filaments to integrins clustered in the 

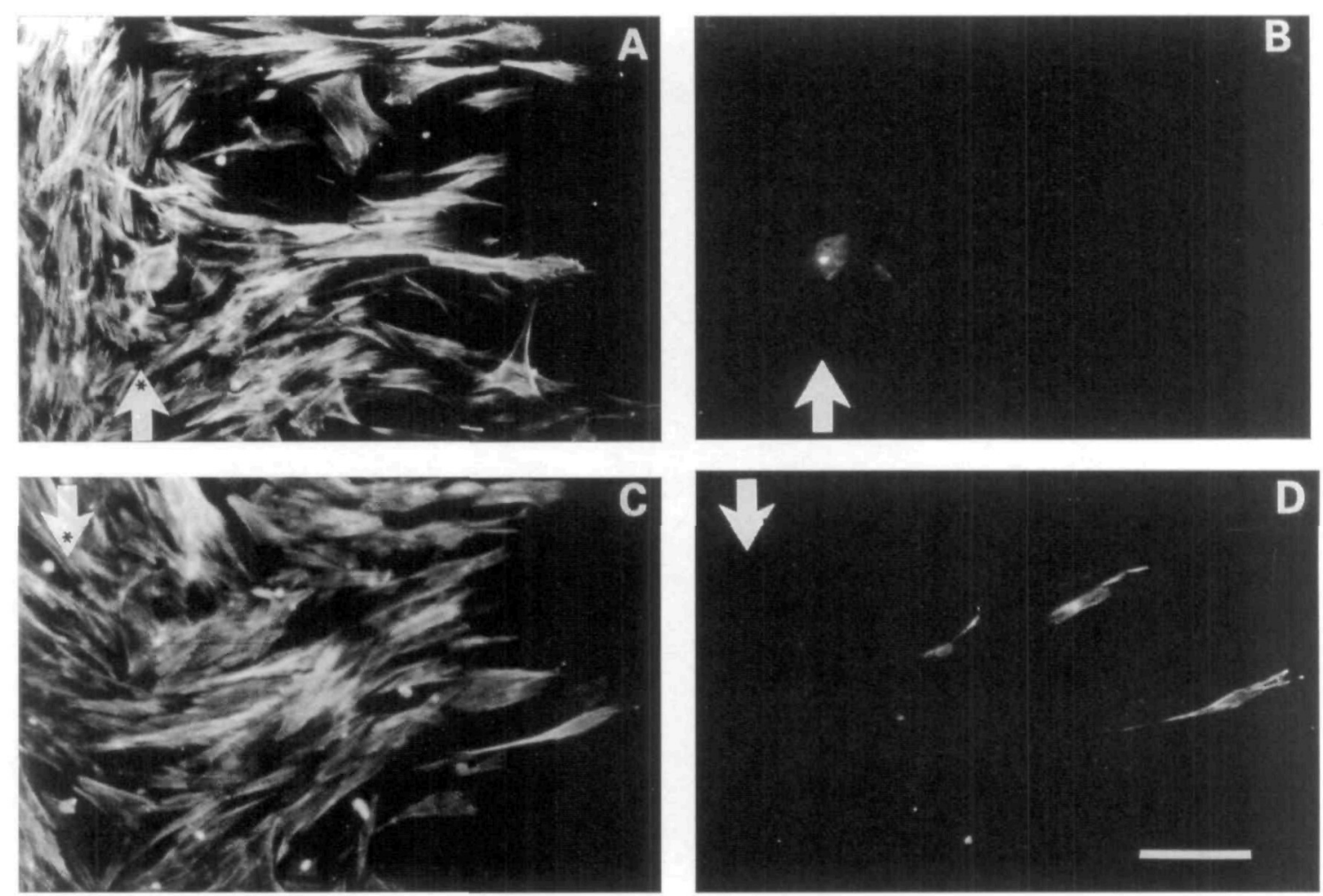

Fig. 7. The effects of anti-talin antibody microinjection on fibroblast migration. CEF were plated onto fibronectin-coated coverslips for $24 \mathrm{~h}$. The cell population was entirely cleared from one half of the coverslip with a cell scraper, and cells immediately contiguous with the wound edge were microinjected with either anti-talin antibody or control antibody. After an additional $12 \mathrm{~h}$ incubation the injected cells were revealed by staining with fluorescein-conjugated anti-rabbit antibody ( $B$ and D), and rhodamine-conjugated phalloidin was used to stain all cells on the coverslip (A and C). Paired images of the same microscopic field are displayed for the anti-talin (A and B) and control antibody (C and D) groups. The baseline of the wound edge is marked (white arrows). The anti-talin-injected cell (B) does not migrate from the wound edge, and is bypassed by noninjected neighboring cells that migrate into the denuded portion of the coverslip (A). Control antibodyinjected cells migrate away from the wound edge (D) as well as their noninjected neighbours (C). Bar, $100 \mu \mathrm{m}$.

plasma membrane. In this paper we have attempted to explore the function of talin by introducing affinitypurified antibodies directed against talin into living fibroblasts cultured on a fibronectin substratum. The effects that we observed suggest that talin is essential for focal adhesion formation and for the maintenance of newly formed adhesions in fibroblasts grown on fibronectin. In fully spread cells with mature focal adhesions, the injected antibody localized to the focal adhesions and fibrillar streaks. However, even with a three hour incubation after antibody injection, the cells remained spread and showed normal adhesions as judged by IRM. In previous studies, the microinjection of antibodies against vinculin failed to cause cell rounding, although a partial loss of stress fibers and focal adhesions was observed (Birchmeier et al., 1982; Jockusch and Fuchtbauer, 1983; Westmeyer et al., 1990). In another study, no noticeable effect on focal adhesions or stress fibers was detected when antibodies against either actin or $\alpha$-actinin were injected into cells (Jockusch and Fuchtbauer, 1983). These previous studies used cells that were fully spread. In contrast, when we introduced anti-talin antibodies into cells that had been freshly plated and that were in the process of spreading and forming new focal adhesions, the spreading was inhibited and a large percentage of these cells were caused to round up. Those cells that rounded up lost focal adhesions and associated stress fibers. In addition, the migration of well-spread fibroblasts with mature focal adhesions was also inhibited by anti-talin antibodies. A small percentage of cells were able to spread out after injection of anti-talin in the cell spreading assay. Similarly, a small percentage of cells injected with anti-talin in the migration assay were able to migrate away from the baseline. We suspect that this represents variability in the amount of antibody introduced into cells, and so immobilization of talin may not have been complete in this small percentage of cells. Our results indicate that talin is required for cells to form new adhesions on fibronectin during cell spreading and during migration.

During the first few minutes of adhesion and spreading, the distribution of talin in fibroblasts appears, by immunofluorescence, to be predominantly as a soluble pool (G.H.N. unpublished results). Detailed analysis of spreading margins of fibroblasts has revealed two distinct sites of talin accumulation (DePasquale and Izzard, 1991). Talin is concentrated in nodes at the tips of F-actin-rich precursors of focal adhesions, along the extreme edge of the spreading 
lamellipodium. Independent of its accumulation in these nodes, talin also concentrates at newly formed sites of adhesion between the substratum and the plasma membrane. The injection of anti-talin into cells that were spreading or migrating caused the formation of immunoprecipitates within the cells. We suspect that this precipitation of talin and its consequent depletion from the soluble pool is the major block to the formation of new focal adhesions. We have no evidence that the antibody is able to interfere with the interaction of talin with other proteins. In this regard, the antibody failed to inhibit the interaction of talin with vinculin in assays in vitro (data not shown).

The recruitment of talin from the soluble pool into newly formed focal adhesions is likely to be a similar process in both the cell spreading assay and the cell migration assay. However, during cell migration, talin in older focal adhesions near the rear of the cell is most likely released into the soluble pool so that it can then be involved in the formation of new adhesions at the leading edge of the cell. The injected antibody may also inhibit migration by binding and crosslinking talin in existing focal adhesions, making these resistant to disassembly as cells migrate.

An unexpected observation was that spreading cells injected with anti-talin antibodies not only were inhibited from further spreading, but frequently were induced to round up. During this cell rounding the cells disassembled focal adhesions that had already formed. These newly formed adhesions thus appeared to be more easily disrupted than the mature adhesions of fully spread cells. The susceptibility of cells to agents such as RGD-containing peptides and antibodies that inhibit the interaction of integrins with their extracellular ligands also decreases as cells remain in culture (Neff et al., 1982; Horwitz et al., 1984; Hayman et al., 1985). This indicates that focal adhesions become more stable with time in culture. Adhesions may be stabilized on the extracellular face by the accumulation of secreted ECM components. Inside the cell, more components may be recruited to focal adhesions, and the complexity with which these proteins interact may increase. In adhesions developed during spreading and migration, talin may be readily exchangeable with the soluble pool, but it is likely to become more stably associated with the focal adhesion as other proteins associate with and crosslink talin. Consequently, the precipitation of talin from the soluble pool may have little effect on talin that has been stably incorporated into a mature adhesion.

A second reason why the anti-talin antibodies have a disruptive effect on immature adhesions but little or no effect on mature adhesions may be that talin plays a more essential role in the developing adhesions. Talin is concentrated at the leading edge of fibroblasts (Burridge and Connell, 1983; Izzard, 1988; DePasquale and Izzard, 1991) where new focal adhesions are formed (Izzard and Lochner, 1980). In mature adhesions parallel linkages between actin and the membrane may exist that are not present in immature adhesions. Consistent with this possibility, other work from this laboratory has demonstrated an interaction between the actin-binding protein $\alpha$-actinin and integrin cytoplasmic domains (Otey et al., 1990b). It has been suggested that $\alpha$-actinin may be more important in bridging between actin filaments and integrins in mature adhesions, whereas talin may be required for the initial formation of the link between the cytoskeleton and the membrane in focal adhesions (Pavalko et al., 1991). Our current data are consistent with such a role for talin.

We thank Dr. Chris Turner and Dr. Fred Payalko for constructive discussions that contributed to the progress of these experiments, and Natalie McLean for technical assistance. This work was supported by NIH grant GM29860.

\section{References}

Belkin, A. M. and Koteliansky, V. E. (1987). Interaction of iodinated vinculin, metavinculin and $\alpha$-actinin with cytoskeletal proteins. FEBS Lett. 220, 291-294.

Birchmeter, W., Libermann, T. A., Imhof, B. A. and Kreis, T. E. (1982). Intracellular and Extracellular Components Involved in the Formation of Ventral Surfaces of Fibroblasts. Cold Spring Harbor Symp. Quant. Biol. 46, 755-767.

Burrldge, K. and Connell, L. (1983). A new protein of adhesion plaques and ruffling membranes. J. Cell Biol. 97, 359-367.

Burridge, K., Fath, K., Kelly, T., Nuckolls, G. and Turner, C. (1988). Focal adhesions: Transmembrane junctions between the extracellular matrix and the cytoskeleton. Annu. Rev. Cell Biol. 4, 487-525.

Burridge, K. and Mangeat, P. (1984). An interaction between vinculin and talin. Nature 308, 744-746.

Couchman, J. R. and Rees, D. A. (1979). The behavior of fibroblasts migrating from chick heart explants: Changes in adhesion, locomotion and growth, and in the distribution of actomyosin and fibronectin. J. Cell Sci. 39, 149-165.

DePasquale, J. A. and Izzard, C. S. (1991). Accumulation of talin in nodes at the edge of the lamellipodium and separate incorporation into adhesion plaques of focal contacts in fibroblasts. J. Cell Biol. 113, 1351-1359.

Duband, J.-L., Nuckolls, G. H., Ishihara, A., Hasegawa, T., Yamada, K. M., Thiery, J. P. and Jacobson, K. (1988). Fibronectin receptor exhibits high lateral mobility in embryonic locomoting cells but is immobile in focal contacts and fibrillar streaks in stationary cells. $J$. Cell Biol. 107, 1385-1396.

Graessmann, A., Graessmann, M. and Mueller, C. (1980). In Methods in Enzymology, vol. 65, pp. 816-825. Academic Press, New York.

Hayman, E. G., Pierschbacher, M. D. and Ruoslahti, E. (1985). Detachment of cells from culture substrate by soluble fibronectin peptides. J. Cell Biol. 100, 1948-1954.

Heath, J. P. and Dunn, G. A. (1978). Cell to substratum contacts of chick fibroblasts and their relation to the microfilament system. A correlated interference-reflexion and high voltage electronmicroscope study. J. Cell Sci. 29, 197-212.

Hock, R. S., Sanger, J. M. and Sanger, J. W. (1989). Talin dynamics in living microinjected nonmuscle cells. Cell Motil. Cytoskel. 14, 271-287.

Horwitz, A., Duggan, K., Buck, C., Beckerle, M. C. and Burridge, K. (1986). Interactions of plasma membrane fibronectin receptor with talin - a transmembrane linkage. Nature 320, 531-533.

Horwitz, A. F., Knudsen, K. A., Damsky, C. H., Decker, C., Buck, C. A. and Neff, N. T. (1984). Adhesion-related integral membrane glycoproteins identified by monoclonal antibodies. In Monoclonal Antibodies and Functional Cell Lines: Progress and Applications (ed. R. H. Kennett, K. B. Bechtol and T. S. McKearn), pp. 103 118. New York: Plenum Press.

Izzard, C. S. (1988). A precursor of the focal contact in cultured fibroblasts. Cell Motil. Cytoskel. 10, 137-142. 
Imzard, C. S. and Lochner, L. R. (1976). Cell-to-substrate contacts in living fibroblasts: an interference reflexion study with an evaluation of the technique. J. Cell Sci. 21, 129-159.

Izzard, C. S. and Lochner, L. R. (1980). Formation of cell-tosubstrate contacts during fibroblast motility: an interference reflection study. J. Cell Sci. 42, 81-116.

Jockusch, B. and Fuchthauer, A. (1983). Organization and function of structural elements in focal contacts of tissue culture cells. Cell Motil. Cytoskel. 3, 391-397.

Kolega, J., Shure, M. S., Chen, W.-T. and Young, N. D. (1982), Rapid cellular translocation is related to close contacts formed between various cultured cells and their substrata. J. Cell Sci. 54, 23-34.

Lemasters, J. J., DiGuiseppi, J., Neiminen, A.-L. and Herman, B. (1987). Blebbing, free $\mathrm{Ca}^{++}$and mitochondrial membrane potential preceding cell death in hepatocytes. Nature 325, 7881.

Molony, L., McCaslin, D., Abernethy, J., Paschal, B. and Burridge, K. (1987). Purification and characterization of talin from chicken gizzard smooth muscle. J. Biol. Chem. 262, 7790-7795.

Muguruma, M., Matsumura, S. and Fukazawa, T. (1990). Direct interaction between talin and actin. Biochem. Biophys. Res. Commun. 171, 1217-1223.

Neff, N. T., Lowrey, C., Decker, C., Tovar, A. and Damsky, C. (1982). A monoclonal antibody detaches embryonic skeletal muscle from extracellular matrices. J. Cell Biol. 95, 654-666.

Nuckolls, G. H., Turner, C. E. and Burridge, K. (1990). Functional studies of the domains of talin. J. Cell Biol. 110, 1635-1644.

Otey, C., Griffiths, W. and Burridge, K. (1990a). Characterization of monoclonal antibodies to chicken gizzard talin. Hybridoma 9, 5762.

Otey, C. A., Pavalko, F. M. and Burridge, K. (1990b). An interaction between a actinin and the $\beta_{1}$ integrin subunit in vitro. J. Cell Biol. 111, 721-729.

Otto, J. J. (1983). Detection of vinculin-binding proteins with an ${ }^{125} \mathrm{I}$. vinculin gel overlay technique. J. Cell Biol. 97, 1283-1287.

Pavalko, F. M., Otey, C. A., Simon, K. O. and Burrtdge, K. (1991) $\alpha$-Actinin: a direct link between actin and integrins. Biochem. Soc. Trans. 19, 1065-1069.

Rochlin, M. W., Chen, Q. M., Tobler, M., Turner, C. E., Burridge, K. and Peng, H. B. (1989). The Relationship Between Talin and Acetylcholine Receptor Clusters in Xenopus Muscle Cells. J. Cell Sci. 92, 461-472.

Wachsstock, D. H., Wilkins, J. A. and Lin, S. (1987). Specific interaction of vinculin with $\alpha$-actinin. Biochem. Biophys. Res. Commun. 146, 554-560.

Westmeyer, A., Ruhnau, K., Wegner, A. and Jockusch, B. M. (1990). Antibody Mapping of Functional Domains in Vinculin. EMBO J. 9, 2071-2078.

Wilkins, J. A., Chen, K. Y. and Lin, S. (1983). Detection of high molecular weight vinculin binding proteins in muscle and nonmuscle tissues with an electroblot-overlay technique. Biochem. Biophys. Res. Commun. 116, 1026-1032.

Wilkins, J. A., Risinger, M. A., Coffey, E. and Lin, S. (1987) Purification of a vinculin binding protein from smooth muscle. $J$. Cell Biol. 105, 130a.

(Received 21 November 1991 - Accepted, in revised form, 20 April 1992) 